\title{
Study on Three-dimensional Structures in Injection-molded iPP/Poly(ethylene-co-octene) by Transmission Electron Microtomography
}

\author{
Michio ONO ${ }^{1,4, *}$, Hideo NishiOKA ${ }^{2}$, Hiroshi JinNAI ${ }^{3}$, Ken NAKAJIMA ${ }^{4}$ and \\ Toshio NisHI ${ }^{4}$ \\ ${ }^{1}$ Kawasaki Development Center, SunAllomer Ltd., \\ 2-3-2 Yako-cho, Kawasaki-ku, Kawasaki, Kanagawa 210-0863, Japan \\ ${ }^{2}$ Joint Research Center for Project on Nanostructure Polymeric Materials, Japan Chemical Innovation Institute (JCII), \\ Kyoto Institute of Technology, Kyoto 606-8585, Japan \\ ${ }^{3}$ Department of Polymer Science and Engineering, Kyoto Institute of Technology, \\ Matsugasaki, Sakyo-ku, Kyoto 606-8585, Japan \\ ${ }^{4}$ Department of Organic and Polymeric Materials, School of Science and Engineering, Tokyo Institute of Technology, \\ 1-12-1, Ookayama, Meguro-ku, Tokyo 152-8552, Japan \\ *Corresponding author: Michio_Ono@sunallomer.co.jp \\ Received November 22, 2005; Revised March 27, 2006; 2nd Revised June 19, 2006; 3rd Revised June 23, \\ 2006; Accepted August 7, 2006 \\ (C) 2006 The Society of Rubber Industry, Japan
}

\begin{abstract}
A phase-separated structure of the injection-molded isotactic polypropylene (iPP)/poly(ethylene-co-octene) (EOR) binary blend was studied in three-dimension (3D) by transmission electron microtomography (TEMT). Highly oriented EOR domains along both flow- (FD) and transverse-to-flow (TD) directions resulting in stacking lamella-sheet like structures to normal direction (ND) were confirmed. Some irregularities in morphology and intervals between the EOR sheets, and thickness heterogeneity of the sheets, were observed more frequently in the TD rather than in the FD. Using the 3D information obtained by the TEMT, we have tried to elucidate massive anisotropy in linear thermal expansion coefficient (CLTE) along the injection directions in this blend. We found that the CLTE anisotropy was well correlated with the lamella-like sheets arrays and their irregularities.
\end{abstract}

Keywords Transmission electron microscopy, Transmission electron microtomography, Isotactic polypropylene, Thermoplastic elastomer, Linear thermal expansion coefficient.

\section{Introduction}

The amount of isotactic polypropylene (iPP) in the automobile industry has been increasing spectacularly. It is used for injection-molded parts such as bumper fascia, dashboard, instrument panel, door trim and pillar garnish ${ }^{1}$. These parts are usually subject to severe thermal conditions ranging from 223 to $393 \mathrm{~K}$. Hence, the accurate dimensional stability toward temperature change is required. It has been well known that the iPP has a high thermal expansion coefficient of $1.4 \times 10^{-4}-1.5 \times 10^{-5}$ $\mathrm{K}^{-12)}$. Therefore, a gap between the iPP-based part and the parts consisting of metal or other polymers arises due to the large difference for the coefficient of linear thermal expansion (CLTE) of metal $\left(1.0 \times 10^{-6}-3.0 \times 10^{-6} \mathrm{~K}^{-1}\right)^{3)}$ and $\mathrm{PP}\left(1.4 \times 10^{-4}-1.5 \times 10^{-4} \mathrm{~K}^{-1}\right)$.
One of the conventional solutions for such issue is adding inorganic fillers (talc, glass fiber and mica) with high aspect ratios, and suppressing the volume expansion by simple mechanical restraints ${ }^{4-6}$. However, such fillerPP composites have caused poor appearance and weight increase in molded articles fabricated using them.

Recently, new techniques were proposed to reduce the CLTE of the injection-molded iPP-based parts in which thermoplastic elastomers (TPE) having well-controlled structures are used as an additive instead of fillers to tune the CLTE $^{7-10}$. In these papers, the elastomers inclusions were highly deformed both along the FD and transverseto-flow (TD) directions and co-continuously dispersed with the iPP. The CLTE values parallel both to FD and TD suppressed to be very low values, although the CLTE in the thickness direction (ND) substantially increase. Such 
anisotropic CLTE behaviors are considered to be caused by the anisotropic morphologies of the elastomer inclusions.

The TPE morphologies, dominant factor on the CLTE control, were investigated using transmission electron microscopy (TEM) or scanning electron microscopy (SEM) in these papers. However, the images obtained by these methods are just two-dimensional (2D) projections of the 3D morphologies. Hence, there had some uncertainty in discussing the CLTE anisotropy (3D property) on the basis of the TEM or the SEM images on each direction (2D images).

The advent of transmission electron microtomograpy (TEMT) has enabled us to acquire the visual information of the 3D morphologies ${ }^{11,12)}$. TEMT uses a series of TEM micrographs taken at various tilt angles, which are reconstructed on the basis of the computerized tomography (CT) to generate 3D images. Spatial resolution of the TEMT is ca. $1 \mathrm{~nm}$ and the 3D images can be used to calculate quantitatively the "scattering profile" by Fouriertransformation of the images ${ }^{13)}$. Hence it has been applied to investigate the structural analysis for polymeric materials ${ }^{14,15)}$; the network structures for block copolymers ${ }^{16-18)}$ and the nanostructure in polymer/clay nanocomposites $^{19)}$.

In this study, we observed the 3D morphologies by TEMT for the injection-molded iPP/EOR binary blend system, which showed massive anisotropy in the CLTE along each direction ${ }^{8,9)}$. The 3D arrays and morphology of the EOR domains, which were just speculation by the TEM images taken from two cross-sections, were visually confirmed to be the stacking lamella-like sheets. Moreover, we discussed the correlation of the 3D morphology observed by the TEMT with the CLTE anisotropy.

\section{Experimental}

\section{Materials and blend sample preparation}

Isotactic polypropylene (iPP) (kindly donated from SunAllomer) used in this study had zero shear viscosity at $473 \mathrm{~K}$ of $56,000 \mathrm{Pas}, \mathrm{mmmm}$ pentad sequences of $98.7 \%$ determined by ${ }^{13} \mathrm{C}$-NMR, a melting temperature of $438 \mathrm{~K}$ determined by differential scanning calorimetry (DSC) at a scanning rate of $10 \mathrm{~K} / \mathrm{min}$. Poly(ethylene-co-octene) (EOR) (purchased from Dow Chemical) having zero shear viscosity of $450 \mathrm{Pas}$ at $473 \mathrm{~K}$ and melting temperature of $337 \mathrm{~K}$ (broad) by DSC was used as a TPE.

The blend sample consisting of iPP/EOR (70/30 (vol./vol.) in the presence of an anti-oxidant additive $(0.2$ (mass $\%)$ was prepared by a co-rotating twin-screw

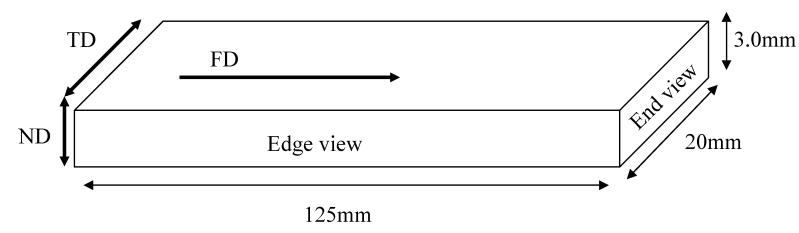

Figure 1. Specimen sizes and definition of the directions and the cross sections; FD, TD and ND represent the flow-, the transverse-toflow-, and the normal directions, respectively. The edge- and end views mean the cross section parallel to FD and TD, respectively.

extruder with L/D of 52 (TEX30 $\alpha$; Japan Steel Works) under cylinder setting temperatures of $453 \mathrm{~K}, 500 \mathrm{rpm}$ of and screw rotation and throughput of $25 \mathrm{~kg} / \mathrm{h}$. It was then fabricated by the injection-molding with an injection machine $(\alpha 100 \mathrm{C}$; Funac) under cylinder setting temperatures of $473 \mathrm{~K}$, full-package time of $2.8 \mathrm{sec}$, and cooling temperature of $313 \mathrm{~K}$ to obtain a slab used in this study.

The slab shape, the directions and the cross-sections used in this study were illustrated in Figure 1; we defined the directions FD as the flow direction, TD as the transverse-to-flow direction and ND as the normal-to-flow direction and the edge view and the end view as the crosssections parallel to FD and parallel to TD, respectively.

The thermal property of the obtained slab, which had been annealed at $373 \mathrm{~K}$ overnight, was measured with DSC ranging from 273 to $503 \mathrm{~K}$. Three endothermic peaks were observed at 311,337 (broad) and $438 \mathrm{~K}$. The peaks at 337 and $438 \mathrm{~K}$ correspond to the melting point $\left(T_{\mathrm{m}}\right)$ of the EOR and the iPP, respectively. The peak at $311 \mathrm{~K}$ would be considered to be the $T_{\mathrm{m}}$ of imperfect EOR crystallites thus thinner lamellae developing upon injection molding or annealing. The EOR molecules with being highly oriented along the flow direction $[8,9$ and the TEMT results in this study] will be more easily crystallized than that without any orientation.

\section{Transmission electron microtomography (TEMT) observation}

The ultra-thin sections for the TEMT measurement with the thickness of ca. $200 \mathrm{~nm}$ were prepared using a cryomicrotome at $173 \mathrm{~K}$, and then stained with $\mathrm{RuO}_{4}$ vapor at room temperature for half an hour. The ultra-thin sections were taken from the core layer for the edge view of the slab. Hence, the TD coincided with the thickness direction of the ultra-thin section.

The TEMT observations for the edge view were carried out on a JEM2200FS (JEOL) operated at $200 \mathrm{kV}^{20}$. Elastically scattered electrons from the ultra thin section 
were used to obtain TEM images through a magnetic prism ( $\Omega$ filter). This enables us to obtain chromatic aberration-free images and thus high contrast and distinctive TEM images were obtained even at high tilt angles. For the $3 \mathrm{D}$ reconstruction, the $2 \mathrm{D}$ TEM images at various tilt angles ranging from $-58^{\circ}$ to $60^{\circ}$ in $1^{\circ}$ increment were taken by a CCD camera $(2048 \times 2048$ pixels) (US1000; Gatan), and then reconstructed by using a software 'Tomograpy' (JEOL). The 3D-images were presented in volume-rendered fashion.

Note that the ideal $180^{\circ}$ range of tilt angles is required to give a perfect 3D image reconstruction. Because reconstructing the $3 \mathrm{D}$ image using $2 \mathrm{D}$ projection images taken at limited tilt angles would result in a loss of resolution and generating ghost images which never exist in the original image. To avoid them, we applied the latest signal reconstruction technique for the 3D image reconstruction in the present study, which decrease the ghost images, thus giving a high contrast 3D image ${ }^{21)}$.

In addition, during tilting the specimen, misalignment in the digitized images are also inevitable due to the imperfect eucentricity of the specimen stage. Hence, alignment of the digitized images is necessary, which was carried out by sequential cross-correlation of the projection images obtained in this study.

\section{CLTE evaluation}

Specimens for the CLTE measurements were cut from the central part of the slab; the size of the specimen was 12 $\mathrm{mm}$ (length) by $5.0 \mathrm{~mm}$ (depth) by $3.0 \mathrm{~mm}$ (width) for FD and TD and $3.0 \mathrm{~mm}$ (length) by $5.0 \mathrm{~mm}$ (depth) by $3.0 \mathrm{~mm}$ (width) for ND. Each specimen was prepared so as the "length" direction can coincide with the FD, TD or ND. Note that the cross section of the specimens was $5.0 \mathrm{~mm}$ (depth) by $3.0 \mathrm{~mm}$ (width) in all cases. The specimens were then annealed at $373 \mathrm{~K}$ for $24 \mathrm{hrs}$ to remove their thermal history and residual stress and to enhance secondary crystallization of the iPP. The measurement was performed in the compression mode using a thermomechanical analyzer (TMA) (MTS9000; Sinku Riko) from 173 to $373 \mathrm{~K}$ at a heating rate of $4.0 \mathrm{~K} / \mathrm{min}$. The applied load was $0.039 \mathrm{~N}$, being sufficient to ensure that the probe remains in contact with the sample and small enough to allow the compression strain to be neglected. The CLTE was monitored as a function of temperature in three directions, FD, TD and ND. Each measurement was independently made on a different specimen.

The CLTE $\left(\alpha_{J}\right)$ is expressed as equation (1)

$$
\alpha_{J}=\frac{1}{L_{J, r}}\left(\frac{\partial L_{J}}{\partial T}\right)_{P}
$$

where the subscript $J$ means the directions, FD, TD and ND. $L_{J, r}$ is the length of $J$ direction at a reference temperature (296 K in this work), $T$ is the temperature and $P$ is pressure (atmospheric pressure in this study, thus considered to be constant throughout the measurement).

\section{Results and Discussion}

\section{TEMT observation}

Figures 2(a), (b) and (c) show the 3D image obtained from the edge view; (a) the snap shot at low magnification chosen from the movie, (b) the snap shot at high

(a)

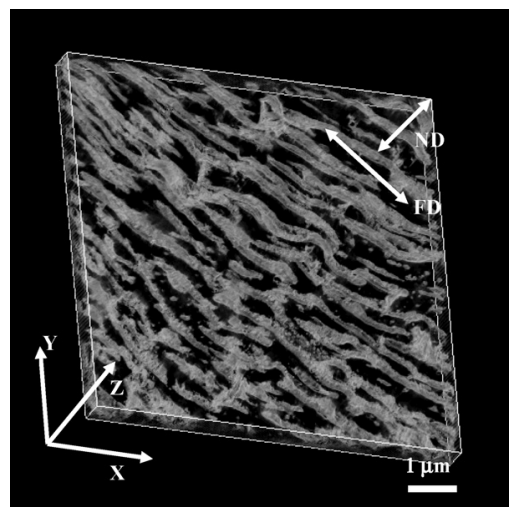

(b)

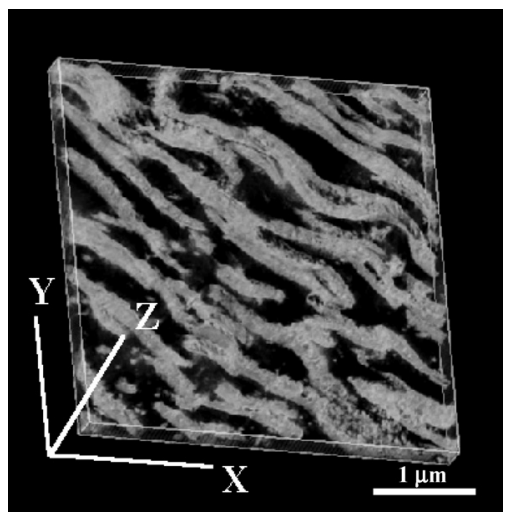

Figure 2. 3D images reconstructed as a volume-rendering; (a) the snap shot taken from the movie at low magnification and (b) the snap shot taken from the movie at high magnification. The frames in the Figures 2(a) and (b) are shown in reconstructed perspective geometry, length (X-axis), width (Y-axis) and thickness (Z-axis). The Z-axis frame in Figure 2(b) is $277 \mathrm{~nm}$ which coincides with the thickness of the ultra-thin section. The arrows in the Figure 2(a) indicate the flow direction (FD) and also the normal (ND) direction. The Z-axis is taken parallel to the TD, the definition of which is applied to the other Figures. Complete sets of the images are shown in forms of animation, which you can find in the electronic appendices. 
magnification from the movie and (c) the movie at high magnification, respectively. The bright regions and the dark regions show the EOR-rich and the iPP-rich phase, respectively. The contrast is inverted from the original TEMT images. The definition of the axes X, Y, and Z used in the TEMT measurement is also designated in Figure 2(a). The Z-axis is taken parallel to the TD. The FD and the ND were also designated in Figure 2(a).

As shown in Figure 2(a), the EOR inclusions are dispersed as co-continuous with the iPP matrix in the XYplane and also highly deformed along the FD.

It is found that, in the XY plane, the EOR domains are highly oriented along the FD and they stack periodically along the ND. Specifically observing the Figure 2(b), the EO domains appear to be oriented along the $\mathrm{Z}$ axis direction, namely to the TD. This can be more clearly confirmed by the movie (Figure 2(c)). The overall morphology of the EOR sheets along the TD requires the EOR sheets information outside the Z-axis frame. Combining the 2D-TEM picture from the end view (Figure 3 ) with the $3 \mathrm{D}$ pictures (Figure 2(a) and (b)), the EOR domains outside the Z-axis frame would be expected to be also highly deformed along the TD as revealed that in Figure 3, most of the EOR inclusions have fibril structures having much longer length than that in the $\mathrm{Z}$-axis frame.

These results suggest that the EOR domains are periodically arranged as the lamella-like sheets stacking along the ND. Such EOR arrays were more firmly speculated by the TEMT method in combination with the 2D-TEM from the end view, compared with the speculation based on the 2D-TEM pictures observed from the two cross-sections, the edge- and end view ${ }^{8,9)}$.

In the XY-plane, the lamella-like sheets of the EOR domains have relatively regular intervals between the sheets and constant thickness of the sheets, indicating that the EOR domains in the FD are relatively regularly arranged.

The YZ- and XZ-slice images provide information about the more detailed internal structures of the TD. The YZslice image (Figure 4(a)) represents a view chosen from the YZ-plane movie moving along the X-direction. The $\mathrm{XZ}$-slice image (Figure 4(b)) is the selected views from the XZ-plane movie along the Y-direction. In these figures, the EOR-rich phases (white regions) appear as a stripe pattern.

In Figure 4(a), the white stripes appear at relatively regular intervals and widths, indicating that the EOR sheets are regularly arranged in the YZ-plane. However, distorted and/or thick EOR phases, the irregular intervals between the EOR sheets are often observed in the XZplane (Figure 4(b)). In addition, another slice image (Figure 4(c)), a selected view of oblique cross-section along the flow direction obtained from the movie,

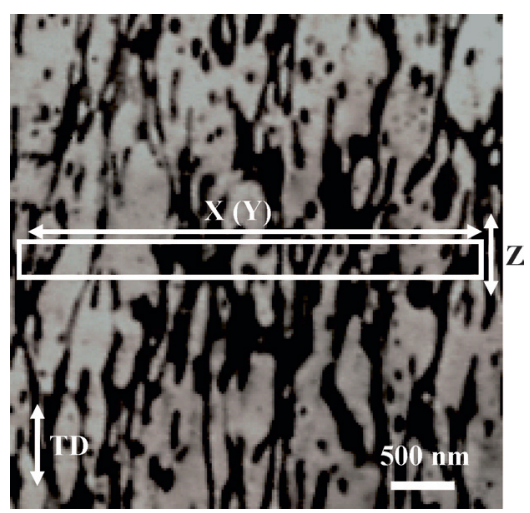

Figure 3. End-view image by 2D-TEM. The frame shown in the Figure represents XZ- or YZ- cross section.

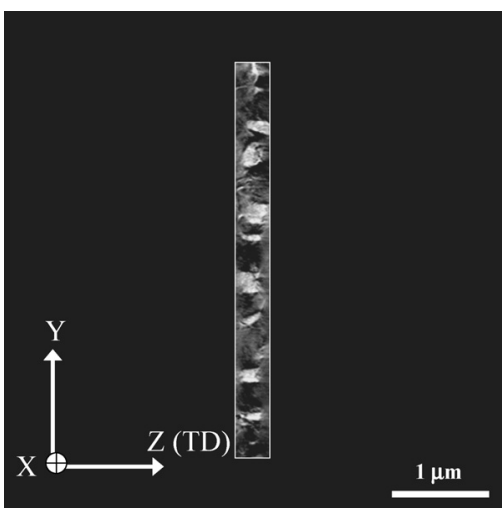

(a)

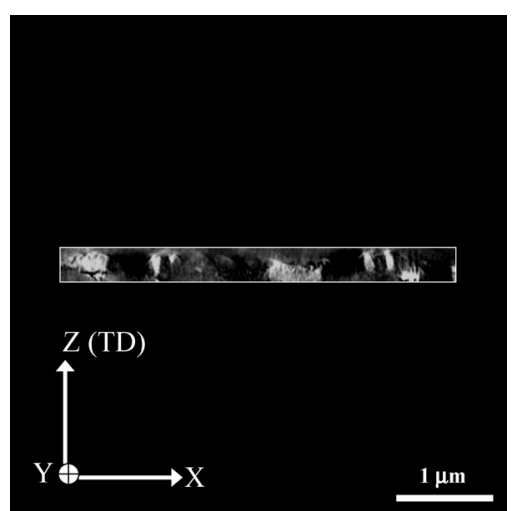

(b)

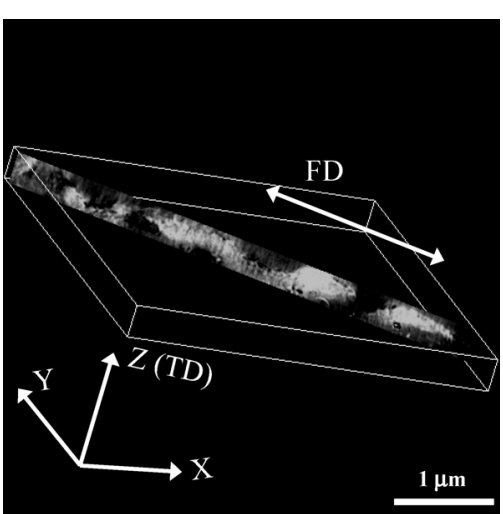

(c)

Figure 4. TEMT slice images; (a) the image chosen from the movie in the XY-plane moving along the Z-axis, (b) the image from the movie in the YZ-plane moving along the $\mathrm{X}$-axis and (c) the image from the oblique slice movie moved perpendicularly to the FD. 
demonstrated that the EOR domains appeared as wavy band structures.

These results suggest that the lamella-like sheets of the EOR domains don't always have a uniform shape over the entire region. Such irregularities tend to be observed more frequently in the entire TD, considering that large amount of irregular structures were observed in the EOR sheets in the TD even within the Z-axis framework. By introducing the irregular EOR sheet structures observed in the TD, the schematic diagram of the EOR morphology is shown in Figure 5.

In general, shear rates, temperatures and pressures applied upon molded articles will not be uniform in the entire region during injection-molding and they will be dependent on the distance from the gate and the depth from the surface ${ }^{22,23)}$. It will induce the morphological heterogeneity; hence the structural irregularities on the lamella-sheets of the EOR domains. In addition, the principal normal stress, making the EOR domains orient along the TD, tend to be much stronger than the shear stress operating along the FD upon injection-molding, which would generate more short-fibrils and/or coarse fibrils frequently observed in the TD as a result of the completion of the fibril-to-droplet transition ${ }^{24,25)}$.

\section{Correlation of CLTE anisotropy with irregularities in the EOR lamella-like sheets}

Figure 6 shows the CLTE profiles on (a) FD, (b) TD, and (c) ND as a function of temperature. We can clearly observe large anisotropy in the overall values of CLTE depending on the directions, i.e., $\alpha_{\mathrm{ND}} \gg \alpha_{\mathrm{TD}}>\alpha_{\mathrm{FD}}$. We have previously pointed out that the driving forces to generate such a massive anisotropy were 1) arrangement of the EOR domains with lamella-like sheets, 2) retraction of the elastomers with being highly deformed and cocontinuous with the iPP ${ }^{10)}$ and 3) PP crystal orientation ${ }^{9}$. In this study, we focused on the investigation of the influence of the former two factors on the CLTE anisotropy.

As demonstrated in Figure 5, the EOR domains with the lamella-like sheets were arranged in parallel to both the FD and TD while in series to the ND. Such arrangement may result in large CLTE in the ND because the total thermal strain in the ND is the summation of that of each lamella-like sheet. In addition, the retraction of highly oriented EOR along both the FD and TD is effective in suppressing the thermal expansion in both the FD and TD while in enhancing the thermal expansion on the ND.

In Figure 6, the CLTE values in the FD and TD drop off sharply above $308 \mathrm{~K}$ ( $T_{\mathrm{m}}$ of the EOR in the blend) whereas

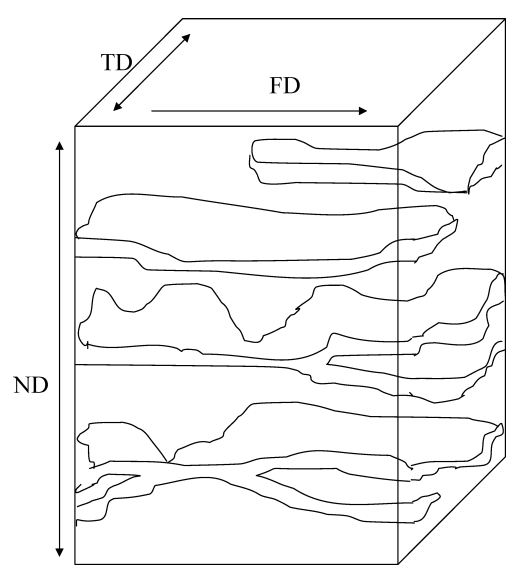

Figure 5. Schematic arrays of the lamella-like sheets in the EOR domains by introducing the irregular morphology frequently found in the TD.

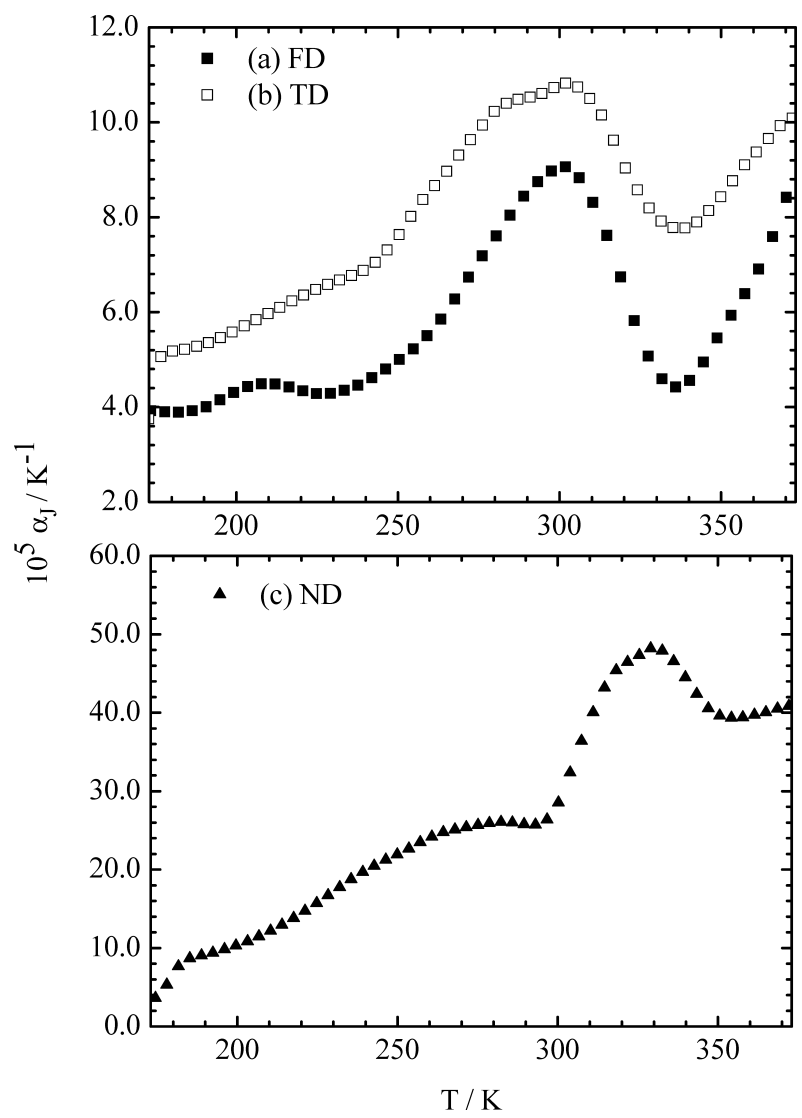

Figure 6. CLTE profiles as a function of temperature on (a) FD, (b) TD and (c) ND.

the CLTE in the ND shows a sudden upturn at this temperature, thus indicating that the lamella-like sheets arrays and the retraction by the interfacial tension of the molten-state EOR inclusions will have correlation with the CLTE anisotropies on the injection directions. 
In addition, the CLTE in the TD above $308 \mathrm{~K}$ tends to decrease more gently than in the FD, which would be ascribed to the irregularities of the thickness of the EOR sheets, namely wider distribution of the aspect ratio of the EOR sheets. The EOR sheets having small aspect ratio (thick EOR sheets) would less contribute to the suppression of the oriented EOR domains by the retraction of the molten-state EOR sheets.

Furthermore, the irregularities of the lamella-like sheets frequently observed in the TD can somewhat account for the CLTE differences in the TD and FD, i.e., $\alpha_{\mathrm{TD}}>\alpha_{\mathrm{FD}}$. From 173 to $308 \mathrm{~K}$, the CLTE in the TD always show higher value compared to that in the FD and the peak at $218 \mathrm{~K}$ ( $T_{\mathrm{g}}$ of the EOR in the blend) is clearly observed in the CLTE profile in the FD while it is not found in the TD. That would, in part, result from more frequent irregularities of the arrangement of the lamella-like sheets observed in the TD, namely wider distribution of the EOR sheet arrays in the TD than that in the FD.

Using the TEMT method, we could investigate the influence of the arrays and the irregularities of the EOR domains on the CLTE anisotropy in detail. However, in addition to them, the other factors, iPP crystal orientation ${ }^{9)}$ and the tie molecules pulled out from the iPP crystalline region $^{26,27)}$ will also be deeply connected with this anisotropic CLTE phenomenon. We will now carry out the analysis of the CLTE anisotropy by introducing these factors together with the TEMT results obtained in this study. It will be reported in the future.

\section{Concluding remarks}

In this study, we presented the $3 \mathrm{D}$ morphologies for the phase-separated structures of the injection-molded iPP/TPE (EOR) binary blend. Combining the TEMT method and 2D-TEM, we confirmed that the EOR domains were highly deformed to FD as well as TD, thus forming the lamella-like sheets stacked parallel to ND, and were also dispersed co-continuously with the matrix iPP. According to the YZ-, XZ-images and the obliquely sliced image, the EOR lamellae-sheets didn't always have uniform shape over the entire region but some irregular structures; distorted shape, irregular thickness and nonuniform intervals between the EOR lamella-sheets, which were expected to be pronounced in the EOR lamella-like sheets in the TD. The presence of such irregularities caused, to some extent, the CLTE anisotropy, especially the difference for $\alpha_{\mathrm{TD}}>\alpha_{\mathrm{FD}}$.

\section{References}

1) Iwano M., Yasuda T., Kusakawa N.: "Zidousyayo Purasuchik", Kogyo Chousakai, Tokyo (1997).

2) Bandrup J., Immergut E. H.: "Polymer Handbook", 3rd ed., Wiley, New York (1989).

3) Ushigi H., Sato T., Hashitani T.: "Kagaku Binran Kisohen II", Maruzen, Tokyo (1993), p. 17.

4) Yoon P. J., Fornes T. D., Paul D. R.: Polymer, 43, 6727 (2002).

5) Holliday L., Robinson J.: J. Mater. Sci., 8, 301 (1973).

6) Kikuchi H., Koyama K.: Seikei-Kakou, 5, 129 (1995).

7) Nomura T., Nishio T., Taniguchi H., Hirai I., Hisamura N.: Kobunshi Ronbunshu, 51, 505 (1994).

8) Ono M., Washiyama J., Nakajima K., Nishi T.: Polym. J., 36, 563 (2004).

9) Ono M., Washiyama J., Nakajima K., Nishi T.: Polymer, 46, 4899 (2005).

10) Wu G., Nishida K., Takagi K., Sano H., Yui H.: Polymer, 45, 3085 (2004).

11) Frank J.: "Electron Tomography: Three-dimensional Imaging with the Transmission Electron Microscope", Plenum Press, New York (1992).

12) Midgley P. A., Weyland M.: Ultramicroscopy, 96, 413 (2003).

13) Jinnai H.: "Interface in Rubber and Elastomer", Ed. Nishi T., CMC Publishing, Tokyo (2003) p. 50.

14) Jinnai H., Nishilkawa Y., Nishi T.: Kobunshi Ronbunshu, 62, 488 (2005).

15) Jinnai H., Nishikawa Y., Ikehara T., Nishi T.: Adv. Polym. Sci., 170, 115 (2004).

16) Spontak R. J., Fung J. C., Braunfeild M. B., Sedat J. W., Agard D. A., Kane L., Smith S. D., Satkowski M. M., Ashraf A., Hajduk D. A., Gruner S. M.: Macromolecules, 29, 4494 (1996).

17) Spontak R. J., Williams M. C., Agard D. A.: Polymer, 29, 387 (1988).

18) Yamaguchi K., Takahashi K., Hasegawa H., Iatrou H., Hadjichristidis N., Kaneko T., Furukawa Y.: Macromolecules, 36, 6962 (2003).

19) Nishioka H., Niihara K., Keneko T., Yamanaka J., Inoue T., Nishikawa Y., Nishi T., Jinnai H.: Composite Interface, in press.

20) Nishikawa Y., Jinnai H., Furukawa H., Naruse M.: Kinozairyo, 22, 11 (2002).

21) Saito T.: "Gazou Shori Arugorizumu", Kindai Kogakusha, Tokyo (1993).

22) Jang B. Z., Uhlmann D. R., Vander Sande J. B.: Polym. Eng. Sci., 25, 64 (1985).

23) Karger-Kocsis J., Cskai I.: Polym. Eng. Sci., 27, 241 (1987).

24) Vadas E. B., Goldsmith H. L., Mason S. G.: Trans. Soc. Rheol., 20, 373 (1976).

25) Han C. D., Kim Y. W.: Trans. Soc. Rheol., 19, 245 (1975).

26) Choy C. L., Chen F. C., Young K.: J. Polym. Sci. Polym. Phys. Ed., 19, 335 (1981).

27) Choy C. L., Chen F. C., Ong E. L.: Polymer, 20, 1191 (1979). 